chest radiograph showed complete reexpansion of the lung. Once at the hospitalization unit, the patient started oral intake and walking; he was discharged home with a new chest radiograph 8 hours after the surgery showing no complications. He resumed normal activities the day after the surgery without any mishaps.

The final pathologic examination reported nasopharyngeal carcinoma metastasis (14 and $7 \mathrm{~mm}$, respectively) with a free margin of $0.9 \mathrm{~cm}$.

\section{DISCUSSION}

Thoracic surgery is evolving toward less invasive approaches. In recent years, uniportal VATS (single port) has gained approval in many centers, ${ }^{1}$ seeking reduction in postoperative pain compared with traditional VATS, even though there is still a lack of clinical trials comparing both approaches.

Many studies have shown the advantages of avoiding general anesthesia and orotracheal single-lumen intubation with mechanical ventilation, thus potentially decreasing the risk of postoperative complications. ${ }^{2}$ Several groups have also examined the safety and feasibility of performing pulmonary resections, such as metastasectomies and even lobectomies, in awake patients. ${ }^{3}$ The surgical pneumothorax created with the incision, specially with a uniportal incision, provides excellent lung collapse; thus, adjunctive measures such as endobronchial blockers are not necessary in most cases.

Our patient was carefully selected because his past history of chemoradiotherapy in the neck made doublelumen intubation more difficult. Surgical pneumothorax leads to mild hypoxemia and hypercapnia usually develops, but in most cases is tolerable to the patient. ${ }^{4}$ Epidural anesthesia proved to be an excellent method of thoracic anesthesia, allowing the diaphragm to be mobile, thereby counteracting the effects of paradoxic ventilation and mediastinal shift. Avoiding general anesthesia allows the patient to resume normal activities, such as drinking, eating, and walking, earlier; the patient can even be discharged the same day as the operation, reducing hospitalization time and costs. ${ }^{5}$

In conclusion, we believe that, in selected patients, a uniportal VATS incision combined with an epidural catheter with the patient breathing spontaneously, is a safe and feasible approach for performing pulmonary resections of peripheral metastatic nodules. It also can be incorporated in a fast-track program with the purpose of reducing the hospitalization time and the costs of the procedure. Prospective randomized clinical trials are needed to support this hypothesis.

\section{References}

1. Gonzalez-Rivas D, Paradela M, Fieira E, Velasco C. Single-incision video-assisted thoracoscopic lobectomy: initial results. J Thorac Cardiovasc Surg. 2013;143: $745-7$

2. Noda M, Okada Y, Maeda S, Sado T, Sakurada A, Hoshikawa Y, et al. Is there a benefit of awake thoracoscopic surgery in patients with secondary spontaneous pneumothorax? J Thorac Cardiovasc Surg. 2012;143:613-6.

3. Chen KC, Cheng YJ, Hung MH, Tseng YD, Chen JS. Nonintubated thoracoscopic ling resection: a 3-year experience with 285 cases in a single institution. J Thorac Dis. 2012;4:347-51.

4. Pompeo E, Mineo TC. Awake pulmonary metastasectomy. J Thorac Cardiovasc Surg. 2007;133:960-6.

5. Rocco G, Romano V, Accardo R, Tempesta A, La Manna C, La Rocca A, et al. Awake single-access (uniportal) video-assisted thoracoscopic surgery for peripheral pulmonary nodules in a complete ambulatory setting. Ann Thorac Surg. 2010; $89: 1625-7$

\title{
Aggressive infective endocarditis and the importance of early repeat echocardiographic imaging
}

\author{
Kyle Eudailey, MD, Jennifer Lewey, MD, Rebecca T. Hahn, MD, and Isaac George, MD, New York, NY
}

\footnotetext{
From the Division of Cardiothoracic Surgery and Department of Cardiology, and the Center for Interventional and Vascular Therapy, New York-Presbyterian Hospital, Columbia University Medical Center, and the Cardiovascular Research Foundation, New York, NY.

Disclosures: Authors have nothing to disclose with regard to commercial support.

Received for publication June 6, 2013; revisions received Sept 27, 2013; accepted for publication Oct 28, 2013; available ahead of print Dec 2, 2013.

Address for reprints: Isaac George, MD, Division of Cardiothoracic Surgery, New York-Presbyterian Hospital-Columbia University Medical Center, Milstein Hospital, 7GN-435, 177 Fort Washington Ave, New York, NY 10032 (E-mail: ig2006@columbia.edu).

J Thorac Cardiovasc Surg 2014;147:e26-8

$0022-5223 / \$ 36.00$

Copyright (C) 2014 by The American Association for Thoracic Surgery

http://dx.doi.org/10.1016/j.jtcvs.2013.10.069
}

The critical role of echocardiography in the assessment of suspected or known infective endocarditis (IE) has been well established. We present a report of aggressive aortic valve $(\mathrm{AV}) \mathrm{IE}$ in a 24-year-old man and reinforce the need for repeat echocardiographic imaging after negative results of examination if clinical suspicion remains high. ${ }^{1,2}$ Seven days after negative results of both transthoracic echocardiographic (TTE) and transesophageal echocardiographic (TEE) examinations, our patient had an annular abscess develop, with extensive local destruction necessitating emergency surgical repair. 


\section{CLINICAL SUMMARY}

Our patient was seen for left arm and leg weakness and a 4-day history of fevers, chills, headaches, and myalgias. His medical history was unremarkable. He denied any history of intravenous drug use and any recent dental procedures, abrasions or lacerations, or hospitalizations. Results were negative for HIV and tuberculosis, and he had no history suggestive of an immunocompromised state. On physical examination, the patient was afebrile; however, his left arm and left leg displayed increased tone, proximal muscle weakness, and hyperreflexia. No murmurs were observed on cardiac examination. His workup was notable for leukocytosis of 14,000 cells $/ \mu \mathrm{L}$, magnetic resonance imaging demonstrating multiple right-sided infarcts, and blood cultures showing growth of gram-positive cocci in pairs. Vancomycin (1 g intravenously every 12 hours) and oxacillin ( $2 \mathrm{~g}$ intravenously every 4 hours) were prophylactically started on the basis of the blood cultures.

On hospital day 1, a TTE was performed. This showed mild aortic insufficiency posteriorly, possible bicuspid AV, and suspect thickening of the noncoronary AV leaflet; no vegetations or abscesses were noted. In the setting of high-grade fevers (maximum temperature $40^{\circ} \mathrm{C}$ ), additional TEE imaging was pursued the same day. TEE confirmed a bicuspid AV (dominant left and noncoronary cusps with a diminutive right cusp), mild aortic insufficiency posteriorly with associated thickening of the aortic wall, no vegetations, and no perivalvular extension (Figure 1). Given the lack of vegetations, the lack of further evidence of emboli, the hemodynamic stability, and the absence of signs of heart failure, the decision was made to continue antibiotic therapy, rule out other possible sources, and reconsider surgical intervention after resolution of bacteremia and repeat imaging. Further speciation on hospital day 3 revealed methicillin-resistant Staphylococcus aureus sensitive to vancomycin. The patient's first

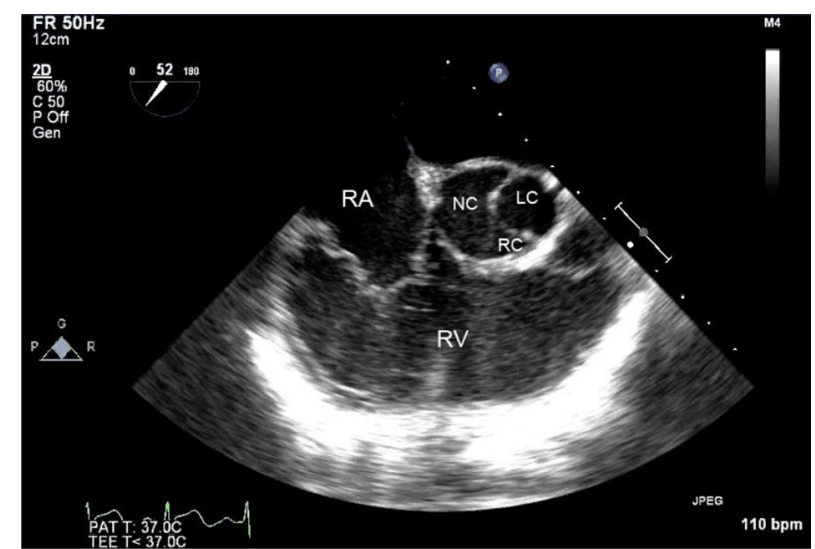

FIGURE 1. Transthoracic echocardiography on hospital day 2 (midesophageal, aortic valve short-axis view). No clear vegetations or abscesses are seen. Dominant noncoronary $(N C)$ and left coronary $(L C)$ cusps are present. $R A$, Right atrium; $R C$, right coronary cusp; $R V$, right ventricle.

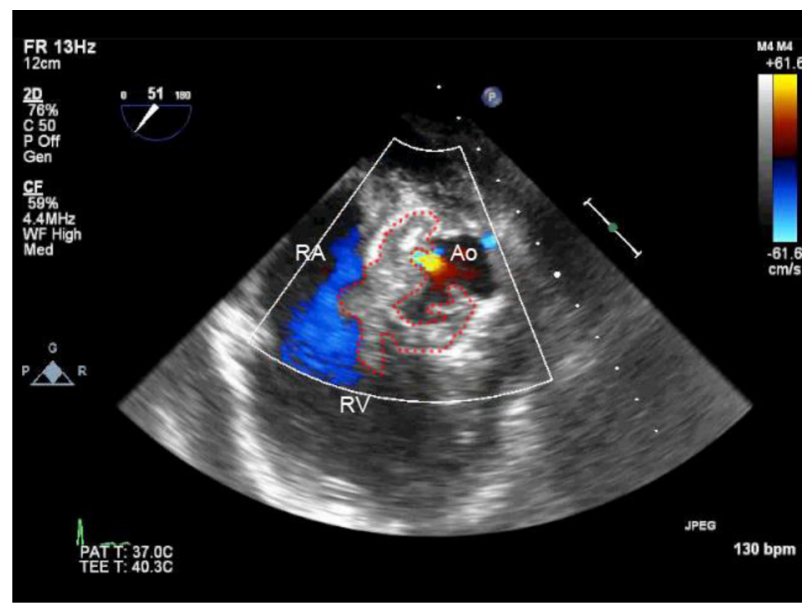

FIGURE 2. Transesophageal echocardiography on hospital day 8 (midesophageal aortic valve short-axis view with color flow). A large vegetation on the noncoronary cusp of the aortic valve, extending to the septal leaflet of the tricuspid valve, is shown (outline). New, severe aortic insufficiency at the noncoronary and left coronary commissure was present. $R A$, Right atrium; $A o$, aorta; $R V$, right ventricle.

vancomycin trough on hospital day 1 was subtherapeutic at $9 \mu \mathrm{g} / \mathrm{mL}$ (goal $>10 \mu \mathrm{g} / \mathrm{mL}$ ), and the dose was increased accordingly. All subsequent levels were therapeutic. Blood culture results remained positive through hospital day 7 , and the patient remained intermittently febrile, although clinically stable and with unchanged symptoms.

On hospital day 8, the patient had acute decompensation in the setting of chest pain, ischemic electrocardiographic changes, and PR interval prolongation. Emergency catheterization showed normal coronary arteries and significantly worsened aortic insufficiency. The patient was taken to the operating room for emergency valve replacement. Antibiotics at this time were broadened to include rifampin (rifampicin, $600 \mathrm{mg}$ intravenously every 24 hours) and gentamicin sulfate (gentamicin, $350 \mathrm{mg}$ intravenously every 24 hours). Preoperative TEE and intraoperative findings showed a $3-\mathrm{cm}$ abscess cavity creating an aorta-right atrial fistula involving the $\mathrm{AV}$ noncoronary sinus and the septal leaflet of the tricuspid valve, as well as destruction of the annulus fibrosa and mitral valve anterior leaflet. Two separate 2 - and $3-\mathrm{cm}$ vegetations were found on the noncoronary cusp of the $\mathrm{AV}$ and the septal leaflets of the tricuspid valve, respectively (Figure 2). A complex repair was completed that encompassed removal of tricuspid valve vegetation, sparing of the septal leaflet, aortic annular débridement, $\mathrm{AV}$ replacement by the Manougian procedure, bovine patch repair of annulus fibrosa and anterior mitral leaflet, and primary repair of the right atrial fistula.

\section{DISCUSSION}

Negative results of echocardiography (both TTE and TEE) in patients with IE are most frequently the result 
of small vegetations $(<3 \mathrm{~mm})$, recent embolization, nonoscillating or atypically located vegetations, or difficult to identify IE lesions in the setting of preexisting cardiac lesions (mitral prolapse, prosthetic valves, intracardiac devices, degenerative or sclerotic valves). ${ }^{3}$ Although the combination of negative TTE and TEE study results provides a $95 \%$ negative predictive value, such results do not definitively rule out vegetative IE. ${ }^{4}$ Current guidelines recommend repeating TEE imaging in 7 to 10 days if clinical suspicion remains high in the setting of an initial negative echocardiographic interpretation. ${ }^{1,2}$ In this report, an aggressive valvular infection with local tissue destruction developed within only 7 days. With a growing body of evidence supporting early surgical intervention in $\mathrm{IE},{ }^{5}$ this case provides a strong justification for repeating echocardiographic imaging, because earlier identification of the severity of infection might have prompted earlier surgical intervention and potentially yielded an improved surgical outcome.

\section{References}

1. Bayer SA, Bolger AF, Taubert KA, Wilson W, Steckelberg J, Karchmer AW, et al. Diagnosis and management of infective endocarditis and its complications. Circulation. 1998;98:2936-48

2. Habib G, Badano L, Tribouilloy C, Vilacosta I, Zamorano JL, Galderisi M, et al Recommendations for the practice of echocardiography in infective endocarditis. Eur J Echocardiogr. 2010;11:202-19.

3. Habib G. Management of infective endocarditis. Heart. 2006:92:124-30.

4. Shively BK, Gurule FT, Roldan CA, Leggett JH, Schiller NB. Diagnostic value of transesophageal compared with transthoracic echocardiography in infective endocarditis. J Am Coll Cardiol. 1991;18:391-7.

5. Kang DH, Kim YJ, Kim SH, Sun BJ, Kim DH, Yun SC, et al. Early surgery versus conventional treatment for infective endocarditis. N Engl J Med. 2012;366:2466-73.

\title{
Ex vivo lung perfusion to evaluate donor lungs after high-pressure pulmonoplegia
}

\author{
Nikhil Prakash Patil, MRCS, MCh, Prashant N. Mohite, MRCS, MCh, André R. Simon, MD, PhD, and \\ Mohamed Amrani, MD, PhD, London, United Kingdom
}

Shortage of organ donors results in significant mortality among patients on transplant waiting lists, necessitating thorough evaluation before declining potentially transplantable organs. We report a case in which donor lung pulmonoplegia was accidentally delivered under high pressure. Despite a potential risk from elevated flushing pressures, the lungs were successfully transplanted after assessment with ex vivo lung perfusion (EVLP). ${ }^{1,2}$

\section{CLINICAL SUMMARY}

Lungs from a 35-year-old female multiorgan donor with brainstem death after intracranial hemorrhage were provisionally accepted for a 52-year-old male patient with pulmonary fibrosis who was on our waiting list. Blood gas values at the time of organ offer showed a ratio of $\mathrm{PaO}_{2}$ to inspired oxygen fraction greater than 300; all other parameters were acceptable.

From the Department of Cardiothoracic Transplantation and Mechanical Circulatory Support, Royal Brompton \& Harefield NHS Foundation Trust, Harefield Hospital, London, United Kingdom.

Disclosures: Authors have nothing to disclose with regard to commercial support.

Received for publication May 15, 2013; revisions received Sept 5, 2013; accepted for publication Sept 30, 2013; available ahead of print Nov 21, 2013.

Address for reprints: Nikhil Prakash Patil, MRCS, MCh, Department of Cardiothoracic Transplantation and Mechanical Support, Royal Brompton \& Harefield NHS Foundation Trust, Harefield Hospital, London, United Kingdom (E-mail: n.patil@rbht.nhs.uk).

J Thorac Cardiovasc Surg 2014;147:e28-9

$0022-5223 / \$ 36.00$

Copyright (c) 2014 by The American Association for Thoracic Surgery

http://dx.doi.org/10.1016/j.jtcvs.2013.09.062
Organ retrieval entailed median sternotomy and satisfactory on-table assessment, including pulmonary vein gas values. After bicaval ligation and aortic clamping, the right atrium and left atrial appendage were incised for venting while the lungs were flushed with $50-\mathrm{mL} / \mathrm{kg}$ cold Perfadex solution (Vitrolife AB, Göteborg, Sweden). Inadvertently, the pulmonary artery was perfused at very high pressures (pressure gauge reading $>150 \mathrm{~mm} \mathrm{Hg}$ ), and the error was noticed only after 2 to 3 minutes (almost all pulmonoplegia delivered). Because of the possibility of alveolocapillary damage in the pulmonary vascular bed, it was decided not to transplant the lungs directly; rather, the donor lungs were evaluated with EVLP to assess suitability for implantation.

After returning to our center, the organs were transferred to the XVIVO Organ Chamber (Vitrolife) and assessed during ex vivo perfusion with STEEN solution (Vitrolife) as described elsewhere. ${ }^{1}$ Briefly, the procedure entails cannulation of the left atrial cuff and main pulmonary artery, tracheal intubation, and ex vivo perfusion with an acellular perfusate under controlled temperature, pressure, and ventilation parameters to mimic physiologic conditions. Both at the beginning and after completion of EVLP, functional parameters such as oxygenation difference (pulmonary vein $\mathrm{PO}_{2}$ - pulmonary artery $\mathrm{PO}_{2}$ ), pulmonary vascular resistance, dynamic lung compliance, and airway pressures were found to be acceptable. Consequently, the lungs were deemed to be suitable for transplantation and implanted with 